\title{
A TYPE OF BORN-INFELD REGULAR GRAVITY AND ITS COSMOLOGICAL CONSEQUENCES *
}

\author{
FRANCO FIORINI ${ }^{\dagger}$ \\ Instituto de Astronomía y Física del Espacio, C.C. 67, Suc. 28, 1428 Buenos Aires, Argentina \\ RAFAEL FERRARO $\ddagger$ \\ Instituto de Astronomía y Física del Espacio, C.C. 67, \\ Suc. 28, 1428 Buenos Aires, Argentina \\ and \\ Departamento de Física, \\ Facultad de Ciencias Exactas y Naturales, \\ Universidad de Buenos Aires, Ciudad Universitaria, \\ Pabellón I, 1428 Buenos Aires, Argentina
}

\begin{abstract}
Born-Infeld deformation strategy to smooth theories having divergent solutions is applied to the teleparallel equivalent of General Relativity. The equivalence between teleparallelism and General Relativity is exploited to obtain a deformed theory of gravity based on second order differential equations, since teleparallel Lagrangian is built just from first derivatives of the vierbein. We show that Born-Infeld teleparallelism cures the initial singularity in a spatially flat FRW universe; moreover, it provides a natural inflationary stage without resorting to an inflaton field. The BornInfeld parameter $\lambda$ bounds the dynamics of Hubble parameter $H(t)$ and establishes a maximum attainable spacetime curvature.
\end{abstract}

\section{INTRODUCTION}

The Born-Infeld (BI) procedure [1] for smoothing singularities is based on the use of a new scale $\lambda$ for introducing the Lagrangian

$$
\mathcal{L} \longrightarrow \mathcal{L}_{B I}=\sqrt{-g} \lambda\left[\sqrt{1+\frac{2 L}{\lambda}}-1\right],
$$

where $\mathcal{L}=\sqrt{-g} L$ is the Lagrangian density whose singularities has to be cured. The scheme (1) is essentially the way for going from the classical free particle Lagrangian to the relativistic one; in such case, the scale is $\lambda=-m c^{2}$, which smoothes the particle velocity by preventing its unlimited growing. In the regime where $L<<\lambda$ the BI Lagrangian (11) becomes the undeformed Lagrangian $\mathcal{L}$. In its original form, the deformation (10) was successfully applied to smooth the divergence characterizing the Coulombian electric field of a point-like charge, and to obtain a finite self-energy for such field configuration.

Inspired by the fruitful properties of the BI program concerning the cure of singularities, we are going to essay a deformation like (11) for the case of a theory of gravity. This issue has deserved some attention in the last years [2, 3, 4, 5, 6, 7]; but in all the cases the research rested on Lagrangians constructed from the Riemann curvature, which unavoidably leads to troublesome fourth order differential equations for the metric. As a matter of fact, explicit solutions for those frameworks (in any dimension) were never found. Following the lines of Ref. [8] we will choose to remain within a second order field equations theory at the price of change the geometrical setting.

In the context of the teleparallel equivalent of General Relativity (TEGR) [9], the dynamical object is not the metric but a set $\left\{e^{a}(x)\right\}$ of four one-forms that turns out to be autoparallel in the Weitzenböck connection $\stackrel{W}{\Gamma}_{\mu \nu}^{\lambda}=e_{a}^{\lambda} \partial_{\nu} e_{\mu}^{a}[10]$. This connection is compatible with the metric $g_{\mu \nu}(x)=\eta_{a b} e_{\mu}^{a}(x) e_{\nu}^{b}(x)$ and curvature free: Weitzenböck spacetime is flat though it possesses torsion $T^{\lambda}{ }_{\mu \nu}=\stackrel{W}{\Gamma}_{\nu \mu}^{\lambda}-\stackrel{W}{\Gamma}_{\mu \nu}^{\lambda}$, which is the agent where the gravitational degrees of freedom are encoded. The TEGR Lagrangian is quadratic in the torsion; the TEGR action with cosmological constant $\Lambda$ reads

$$
\mathcal{I}_{\text {TEGR }}\left[e_{\mu}^{a}\right]=\frac{1}{16 \pi G} \int e(\mathbb{S} \cdot \mathbb{T}-2 \Lambda) d^{4} x, \quad e=\operatorname{det}\left(e_{\mu}^{a}\right)=\sqrt{-g},
$$

\footnotetext{
* Talk given at the 7th Alexander Friedmann International Seminar on Gravitation and Cosmology, Joao Pessoa, Brazil, July 2008.

$\dagger$ franco@iafe.uba.ar

¥ ferraro@iafe.uba.ar. Member of Carrera del Investigador Científico of CONICET.
} 
where $\mathbb{S} \cdot \mathbb{T}=S_{\lambda}^{\mu \nu} T^{\lambda}{ }_{\mu \nu}$, and $\mathbb{S} \equiv S_{\lambda}{ }^{\mu \nu}$ is

$$
S_{\lambda}^{\mu \nu}=-\frac{1}{4}\left(T_{\lambda}^{\mu \nu}-T_{\lambda}^{\nu \mu}-T_{\lambda}^{\mu \nu}\right)+\frac{1}{2} \delta_{\lambda}^{\mu} T_{\theta}^{\theta \nu}-\frac{1}{2} \delta_{\lambda}^{\nu} T_{\theta}^{\theta \mu} .
$$

The dynamical equations resulting from this Lagrangian are equivalent to those of GR for the metric associated with the vierbein [11, 12, 13]. However, the fact that TEGR Lagrangian is built with first derivatives of the vierbein field is very fortunate, because tell us that any deformation of TEGR will still lead to second order equations.

\section{BORN-INFELD GRAVITY AND ITS FRW COSMOLOGICAL SOLUTION}

Hereafter we will call Born-Infeld gravity to the theory obtained from TEGR by means of the following deformation:

$$
\mathcal{I}_{\mathbf{B I}}\left[e_{\mu}^{a}\right]=\frac{\lambda}{16 \pi G} \int e\left[\sqrt{1+\frac{2(\mathbb{S} \cdot \mathbb{T}-2 \Lambda)}{\lambda}}-1\right] d^{4} x
$$

where the $\mathbf{B I}$ scale $\lambda$ has dimensions of inverse squared time.

Replacing the cosmological ansatz $e_{\mu}^{a}=\operatorname{diag}(1, a(t), a(t), a(t))$ (which implies the spatially flat FRW metric $g_{\mu \nu}=$ $\left.\operatorname{diag}\left(1,-a(t)^{2},-a(t)^{2},-a(t)^{2}\right)\right)$ in the Euler-Lagrange equations that result from varying the action $\mathcal{I}_{\mathbf{B I}}+\mathcal{I}_{\text {matter }}$ with respect to the vierbein, we get the motion equations

$$
\begin{gathered}
\frac{1-\epsilon}{\sqrt{1-\epsilon-\frac{12 H^{2}}{\lambda}}}-1=\frac{16 \pi G}{\lambda} \rho(t) \\
\frac{(1-\epsilon)\left(\frac{16 H^{2}}{\lambda}+\frac{8 H^{2} q}{\lambda}-1+\epsilon\right)}{\left(1-\epsilon-\frac{12 H^{2}}{\lambda}\right)^{3 / 2}}+1=\frac{16 \pi G}{\lambda} p(t),
\end{gathered}
$$

where $\epsilon=4 \Lambda \lambda^{-1}$, and $H=\dot{a} a^{-1}$ and $q=-a \ddot{a} \dot{a}^{-2}$ are the Hubble and deceleration parameters respectively. By assuming the fluid state equation $p=\omega \rho$, and using the fluid energy-momentum conservation, which is encoded in Eqs. (5) -(6), it results $\rho(t)=\rho_{o}\left(\frac{a_{o}}{a}\right)^{3(\omega+1)}, \rho_{o}$ and $a_{o}$ being two constants. Replacing $\rho(t)$ and changing to the more convenient variable $\mathrm{y}=\frac{\lambda}{16 \pi G \rho_{o}}\left(\frac{a}{a_{o}}\right)^{3(\omega+1)}$ (for $\omega>-1$ ), Eq. (5) can be easily integrated; the solution for positive cosmological constant $\Lambda$ and $0<\epsilon<1$ is 14]

$$
\mathcal{A} t+c=\ln \left[\frac{\mathrm{y}}{1+\mathrm{y}+\sqrt{1+2 \mathrm{y}+\epsilon \mathrm{y}^{2}}}\right]+\frac{1}{\sqrt{\epsilon}} \ln \left[\frac{1+\epsilon \mathrm{y}}{\sqrt{\epsilon}}+\sqrt{1+2 \mathrm{y}+\epsilon \mathrm{y}^{2}}\right] .
$$

Here $\mathcal{A}=3(1+\omega) \sqrt{\frac{\lambda(1-\epsilon)}{12}}$, and $c$ is an integration constant. The more relevant feature of this solution is that the scale factor behaves as $a(t) \propto \exp \left[\sqrt{\frac{\lambda(1-\epsilon)}{12}} t\right]$ when $\mathrm{y} \rightarrow 0$ (i.e., $a \rightarrow 0$ ). Therefore, the Hubble parameter reaches a maximum value $H_{\max }=\sqrt{\frac{\lambda(1-\epsilon)}{12}}$ at the early stage, curing the physical divergences characterizing GR. This natural inflationary stage is a purely geometrical effect and does not rely on the existence of an inflaton field. In terms of the redshift $z=a_{o} / a(t)-1, H(z)$ becomes a constant when $z$ goes to infinity, implying in this way, that the particle horizon radius $\sigma=a_{o} \int_{0}^{a_{o}}(a \dot{a})^{-1} d a$ diverges. Hence the whole spacetime ends up being causally connected, in agreement with the isotropy of the cosmic microwave background radiation. This fact appears as an essential property of modified teleparallelism which does not require any special assumption about the sources of the gravitational field.

The BI approach (44) generates regular solutions. In the cosmological setting this is so, not only because the scale factor is always different from zero, but because the geometrical invariants (both, in Riemann and Weitzenböck spacetimes) are bounded for any finite values of the cosmological time. In fact, each invariant in Weitzenböck spacetime that is quadratic in the torsion tensor is proportional to $H^{2}$ in the cosmological scenario under consideration. On the other hand, the Riemannian invariants for the metric $g_{\mu \nu}=\operatorname{diag}\left(1,-a(t)^{2},-a(t)^{2},-a(t)^{2}\right)$ can be cast in the polynomical form $\mathcal{P}=(H, \dot{H})$. For instance, the scalar curvature is $R=6\left(2 H^{2}+\dot{H}\right)$, the squared Ricci scalar $R_{\mu \nu}^{2}=R^{\mu \nu} R_{\mu \nu}$ is $R_{\mu \nu}^{2}=12\left(3 H^{4}+3 H^{2} \dot{H}+\dot{H}^{2}\right)$, and the Kretschmann invariant $K=R_{\beta \gamma \delta}^{\alpha} R_{\alpha}^{\beta \gamma \delta}$ reads 
$K=12\left(2 H^{4}+2 H^{2} \dot{H}+\dot{H}^{2}\right)$. All these invariants are well behaved due to the saturation value $H=H_{\max }$ reached by the Hubble parameter as $a(t) \rightarrow 0$. Moreover, a direct calculation shows that these invariants are bounded by the BI parameter $\lambda$, ruling in this way not only the behavior of the inflationary phase, but also establishing a maximum attainable spacetime curvature.

Finally, note that the late time behavior $(\mathrm{y} \rightarrow \infty)$ of the solution of Eq. (7) is $a(t) \propto \exp \left[\sqrt{\frac{\lambda \epsilon(1-\epsilon)}{12}} t\right]$. Since $\epsilon$ should be very small $(\Lambda<<\lambda)$ in order that the theory does not appreciably differ from GR for most of the history of the universe (see in Ref. [8] a lower bound for $\lambda$ ), one concludes that the final stage of the universe is described by $a(t) \sim \exp \left[\sqrt{\frac{\lambda \epsilon}{12}} t\right]=\exp \left[\sqrt{\frac{\Lambda}{3}} t\right]$ as expected, while the initial stage is described by $a(t) \propto \exp \left[\sqrt{\frac{\lambda}{12}} t\right]$. Thus the Born-Infeld scale $\lambda$ would play the role of an effective initial vacuum energy driving the inflationary stage. In this way, the universe evolves from an inflationary stage, driven by the (vacuum-like) energy $\lambda$, to the present $\Lambda$-dominated epoch.

\section{Acknowledgments}

F. Fiorini is indebted to the organizers of the Seventh Alexander Friedmann Seminar for the invitation to participate in this wonderful event held in the very shores of the North Brazilian Atlantic. He also would like to thank G. Dotti, A. Megevand, J. Oliva, D. Tempo and R. Troncoso for the warmth and quality of their conversations during the Seminar. This work was supported by CONICET and UBA.

[1] M. Born and L. Infeld, Proc. R. Soc. A 144, 425 (1934); ibid., 147, 522 (1934); ibid., 150, 141 (1935).

[2] S. Deser and G.W. Gibbons, Class. Q. Grav. 15, 35 (1998).

[3] J. A. Feingenbaum, Phys. Rev. D 58, 124023 (1998).

[4] J. A. Feingenbaum, P.O. Freund and M. Pigli, Phys. Rev. D 57, 4738 (1998).

[5] D. Comelli, Phys. Rev. D 72, 064018 (2005).

[6] D. Comelli and A. Dolgov, JHEP 0411, 062 (2004).

[7] J. A. Nieto, Phys. Rev. D 70, 044042 (2004).

[8] R. Ferraro and F. Fiorini, Phys. Rev. D 75, 084031 (2007).

[9] A. Einstein, Sitzungsber. Preuss. Akad. Wiss., 217 (1928); ibid., 401 (1930); A. Einstein, Math. Annal. 102, 685 (1930). English version in http://www.lrz-muenchen.de/ aunzicker/ae1930.html; F. Gronwald and F. W. Hehl, in Proc. 14th International School on Cosmology and Gravitation, eds. P.G. Bergmann et al. (World Scientific, Singapore, 1996).

[10] R. Weitzenböck, Invarianten Theorie, (Nordhoff, Groningen, 1923).

[11] K. Hayashi and T. Shirafuji, Phys. Rev. D 19, 3524 (1979).

[12] J.W. Maluf, J. Math. Phys. 35, 335 (1994).

[13] H.I. Arcos and J.G. Pereira, Int. J. Mod. Phys. D 13, 2193 (2004).

[14] R. Ferraro and F. Fiorini, Phys. Rev. D 78, 124019 (2008). 